\title{
SUR UN CAS DE GYNANDROMORPHISME NATUREL CHEZ CHIROCEPHALUS DIAPHANUS PREV. \\ [Crustacé, Branchiopode]
}

\author{
par M. Nourisson et R. LENEL.
}

Etudiant la faune des eaux douces temporaires des environs de 'Tunis, nous avons été amenés à observer un cas de gynandromorphisme naturel chez un Chirocephalus diaphanus. Cet unique exemplaire a été reconnu parmi un nombre important de Branchiopodes récoltés dans un fossé longeant le stade municipal d'Ez-Zahra.

On sait que les cas de gynandromorphisme sont relativement fréquents chez Ies Arthropodes. A notre connaissance, en ce qui concerne les Crustacés, de telles aberrations ont été signalées chez Homarus [Herrick 1896], Carcinus maenas [Veillet 1945], Chaetophiloscia sicula [Dalens 1966], Simocephalus sp. [Banta et Coll. 1939], Artemia salina [Bowen et Hanson 1961 ].

Notre observation s'avère d'autant plus intéressante qu'il s'agit du premier cas affirmé de gynandromorphisme naturel chez les Branchiopodes Anostracés. En effet, l'exemple cité par Bowen et Hanson intéresse un individu dont les parents ont été irradiés aux rayons $\mathrm{X}^{1}$.

Avant d'aborder l'étude proprement dite de ce cas de gynandromorphisme, nous rappellerons succinctement quelques caractéristiques de la morphologie externe et interne des individus normaux.

\section{1. - Rappel de la morphologie externe des individus normaux.}

L'espèce Chirocephalus diaphanus présente un dimorphisme sexuel très accusé qui se manifeste essentiellement par la présence d'appendices frontaux chez le mâle et par des différences de structure intéressant les antennes et les segments génitaux.

1. Il convient cependant de signaler que Gisscen, en 1881, a publié un article concemant un Eubranchipus vernalis hermaphrodite. Or, la description qu'il donne de cet individu laisse à penser qu'il s'agit d'un gynandromorphe typique. 
Les antennes du mâle, fortement muscularisées, se composent de deux articles bien développés formant une sorte de pince au moyen de laquelle le mâle maintient la femelle durant l'accouplement. Les antennes de la femelle, en revanche, sont uniarticulées, trapues, légèrement aplaties dorso-ventralement. Les appendices frontaux du mâle, organes pairs insérés à la base des antennes, sont formés d'un appendice serratiforme portant plusieurs petits lobes et une large lame basale. Il n'existe pas d'organes homologues chez la femelle.

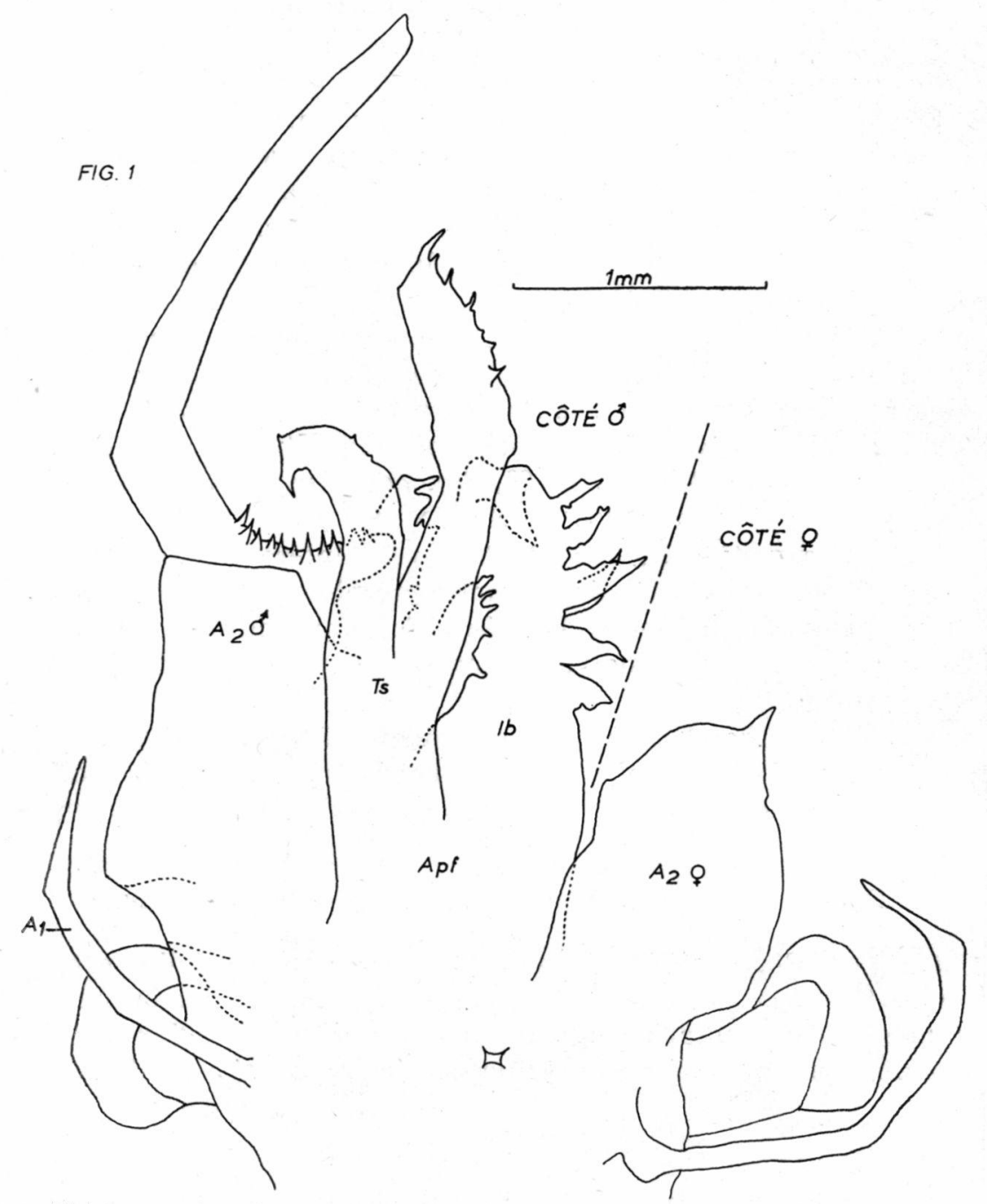

Frg. 1. - Appendices céphaliques (face dorsale).

A1 $=$ antennules; $\mathbf{A 2}=$ antennes $;$ Apf $=$ appendice frontal; $T$ s $=$ tige serratiforme de l'appendice frontal; Ib = lame basale de l'appendice frontal. 
Dans les deux sexes, les deux premiers segments abdominaux portent ventralement une formation impaire. Chez la femelle, c'est une poche oblongue contenant, d'une part l'utérus dans lequel les œufs subissent un début d'incubation, d'autre part un massif de glandes coquillières; cet organe porte le nom de sac ovigère ou de poche incubatricc. Chez le mâle, c'est un simple bourrelet génital orné de deux apophyses chitineuses médianes et pourvu de deux pénis rétractiles.

\section{2. - Rappel de l'anatomie des organes génitaux des individus normaux.}

2.1. - Appareil génital mâle. Les deux testicules sont situés entièrement dans l'abdomen, de part et d'autre du tube digestif. Ils ont l'aspect de tubes plus ou moins rectilignes, légèrement aplatis latéralement. Chaque testicule s'étend approximativement du dernier au troisième segment abdominal et se continue par un spermiducte, puis par un canal déférent qui s'ouvre à l'extrémité d'un pénis rétractile. Le spermiducte communique avec une large vésicule séminale, et, au confluent des deux organes, se trouve un massif de cellules glandulaires géantes à fonction énigmatique. La structure de ces cellules présente des analogies avec celle des glandes coquillières annexées à l'appareil génital femelle.

2.2. - Appareil génital femelle. Comme les testicules, les ovaires sont situés entièrement dans l'abdomen, de part et d'autre du tube digestif. Au niveau du second segment abdominal, chaque ovaire s'ouvre latéro-ventralement dans un oviducte débouchant dans un utérus impair. Cet utérus reçoit les produits de sécrétion des glandes coquillières.

\section{3. - Morphologie externe du gynandromorphe.}

Les appendices céphaliques sont représentés, du côté droit, par une antenne du type femelle, du côté gauche, par une antenne du type mâle et un seul appendice frontal ( $f \mathrm{~g}$. 1).

Les segments génitaux portent ventralement deux formations disposées symétriquement par rapport au plan sagittal de l'animal et totalement indépendantes l'une de l'autre. A droite, il s'agit d'une poche allongéc semblable à un sac ovigère, à gauche c'est un bourrelet génital de type mâle. Chacune de ces formations ne correspond néanmoins qu'à la moitié d'un organe normal; le bourrelet génital mâle, notamment, ne possède qu'un seul pénis et qu'une seule apophyse chitineuse médiane (fig. 2).

Extérieurement, l'individu est donc divisé en deux moitiés latérales dont l'une est d'aspect typiquement mâle et l'autre d'aspect 


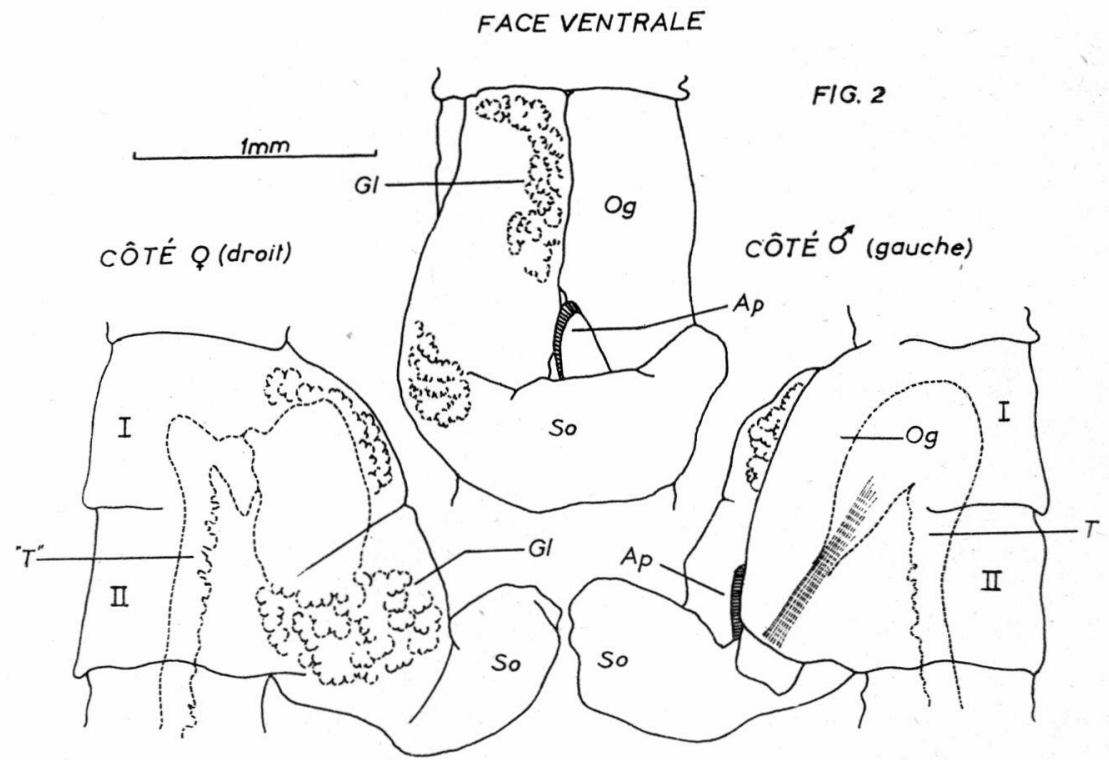

FIG. 2. - Segments génitaux.

I, II $=1^{\text {er }}$ et $2^{\mathrm{e}}$ segments abdominaux $; \mathrm{Gl}=$ glande coquillière; $\mathrm{Og}=$ organe génital externe mâle; $A p=$ apophyse génitale; $\mathrm{So}=\mathrm{sac}$ ovigère; $\mathrm{T}=$ testicule; $\ll \mathrm{T} »=$ gonade femelle masculinisée.

typiquement femelle : il s'agit d'un cas bien caractérisé de gynandromorphisme bilatéral.

\section{4. - Anatomie de l'appareil génital du gynandromorphe.}

L'étude de l'anatomie de l'appareil génital a été faite sur coupes sériées colorées à l'hémalun-éosine. Elle a révélé que ce gynandromorphe possédait deux testicules, siège d'une gamétogenèse en apparence normale. Le tractus génital gauche, c'est-à-dire celui situé dans «la partie mâle» de l'individu, est typique; sa structure correspond parfaitement à celle que nous avons décrite précédemment pour les mâles normaux. En revanche, le tractus génital de «la partie femelle» est aberrant. En effet, le spermiducte qui prolonge le testicule droit se termine en cul de sac. La paroi de sa région terminale affronte celle d'une vaste poche s'ouvrant à l'extérieur par le sac ovigère, mais il n'y a pas de communication franche entre ces deux conduits (fig. 3). Nous avons d'ailleurs remarqué que le spermiducte était rempli de spermatozoïdes alors que cette poche, qui lui est contiguë, en était totalement dépourvue. En outre, ce tractus ne comporte pas de vésicule séminale et le massif glandulaire qui lui est normalement annexé est également 
absent. Précisons que la poche qui fait suite au spermiducte est entourée de gros éléments cellulaires présentant l'aspect caractéristique des glandes coquillières de la femelle : cet organe peut donc être considéré comme un utérus normal.

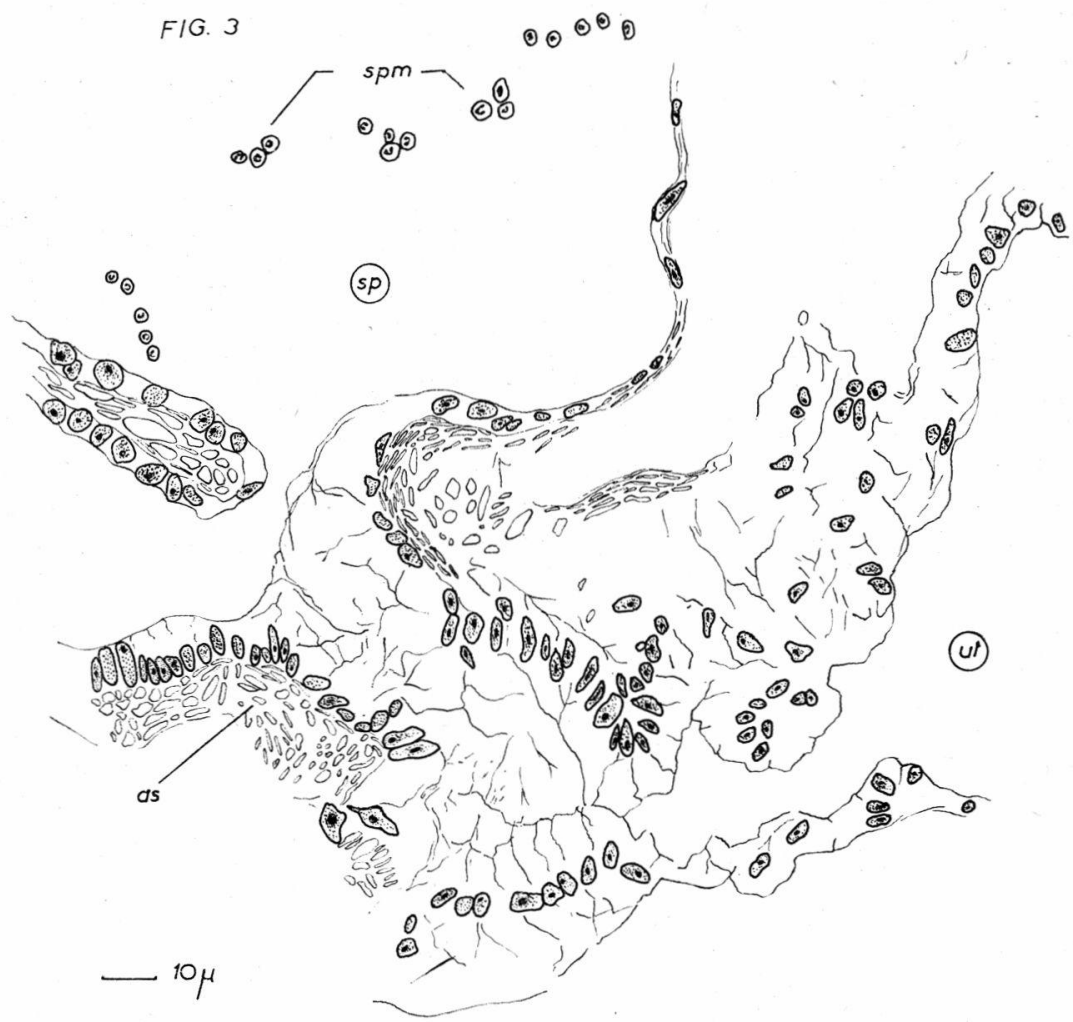

Fig. 3. - Coupe transversale au niveau de la zone de raccordement «spermiducte droit » utérus. On notera l'aspect très particulier des assises épithéliales de cette région. L'hypertrophie de ces tissus entraîne la formation d'un bouchon qui obstrue le passage entre «le spermiducte» et l'utérus.

$\mathrm{sp}=$ lumière du spermiducte; ut = lumière de l'utérus; $\mathrm{spm}=$ spermatozoïdes; as $=$ assise musculaire doublant la paroi du spermiducte.

\section{5. - Discussion et interprétation.}

De nombreuses théories ont été avancées pour expliquer les différents cas de gynandromorphismes (voir notamment BRUsT 1966). En ce qui concerne le cas que nous décrivons ici, nous adopterons l'hypothèse émise par Bowen et HANSON à propos d'un cas de gynandromorphisme chez un autre Branchiopode Anostracé, Artemia salina. Comme ces auteurs, nous supposerons que la partie 
droite et la partie gauche de l'animal ont été déterminées précocement, lors de la première division de segmentation. Dans ces conditions, il est vraisemblable qu'un accident chromosomique entraînant la perte d'un gonosome $\mathrm{X}$ au moment de la première division de l'œuf du type $2 \mathrm{~A}+\mathrm{XX}$ se soit traduit par la formation de deux cellules filles, l'une de type $2 A+X X$, l'autre de type $\mathbf{2 A}+\mathbf{X O}$.

Le problème du déterminisme génétique du sexe chez les Branchiopodes Anostracés a donné lieu à des interprétations diverses. $P_{A I}$ [1949], par exemple, pour Chirocephalus nankinensis, donne le sexe mâle comme sexe hétérogamétique. Bowen [1963], en revanche, a démontré par l'analyse de la transmission de gènes liés au sexe que, chez Artemia salina, c'est la femelle qui est hétérogamétique. La méthode utilisée par ce dernier auteur apporte beaucoup de crédit à son interprétation et, jusqu'à preuve du contraire, nous l'acceptons volontiers pour Chirocephalus diaphanus. Ainsi, nous considérons que «la partie mâle» du gynandromorphe est issue de la cellule normale $2 \mathrm{~A}+\mathrm{XX}$ alors que «la partie femelle » aurait été engendrée à partir de la cellule ayant subi l'accident chromosomique évoqué précédemment.

Quoiqu'il en soit, théoriquement, cet individu devrait être hermaphrodite. Or, l'étude de sa morphologie interne nous a révélé que la gonade de la partie ayant les caractères sexuels externes de type femelle avait histologiquement l'aspect d'un testicule fonctionnel. Il faut donc en conclure que cette gonade, génétiquement femelle, a été masculinisée. Bien que la présence d'un tissu androgène n'ait pas encore été mise en évidence chez les Branchiopodes, on a tout lieu de croire, comme cela a été abondamment démontré chez de nombreux autres Crustacés, que cette masculinisation est due à l'action d'un facteur hormonal androgène issu de «la partie mâle » de l'animal. Toutefois, il importe de souligner que cette inversion sexuelle n'intéresse pas les caractères sexuels externes qui conservent leur aspect typiquement femelle. Les divers territoires, internes et externes, qui, par leur morphologie spécifique, caractérisent le sexe femelle ne présenteraient donc pas la même sensibilité à l'action de l'hormone androgène. Notons à l'appui de notre hypothèse que BALESDENT [1964] a montré expérimentalement que l'hormone androgène était sans action sur les caractères sexuels externes permanents de l'Isopode Asellus aquaticus.

Une étude préliminaire concernant l'organogenèse du tractus génital des individus normaux a permis à l'un de nous de constater que les parties internes (gonades, gonoductes) et les parties externes (organe copulateur, sac ovigère...) de cet appareil se formaient indépendamment l'une de l'autre. Compte tenu de ce qui vient d'être dit au sujet de l'action éventuelle d'un facteur hormonal, 
cela pourrait, d'une certaine manière, expliquer la morphologie aberrante du tractus génital de «la partie femelle». En effet, si l'inversion de la gonade femel!c se produit précocement, on peut supposer que dans la suite du développement ce «testicule »'a pas la possibilité de venir s'aboucher normalement avec la partie externe de l'appareil génital qui, n'ayant pas réagi à l'action de l'hormone androgène, conserve sa différenciation primitive en utérus typique.

\section{RÉSUMÉ}

Dans cette note, les auteurs décrivent un cas de gynandromorphisme bilatéral naturel chez Chirocephalus diaphanus. Ils interprètent cette anomalie sexuelle comme résultant d'un accident chromosomique s'étant produit lors de la première division de segmentation. Ils rejoignent en ccla la théorie que Bowes et Hanson ont formulée à propos d'un cas de gynandromorphisme chez Artemia salina, mais contrairement à ces auteurs, ils admettent la possibilité d'une action hormonale de la partie mâle sur certains tissus de la partie femelle entraînant la masculinisation de la gonade génétiquement femelle.

\section{A CASE OF NATURAL GYNANDROMORPHISM IN CHIROCEPHALUS DIAPHANUS PREV.}

[Crustacea, Branchiopoda]

In this text, the authors describe a case of bilateral gynandromorphism which is natural in Chirocephalus diaphanus. They interpret this sexual anomaly as a result of a chromosomic accident which took place at the first division of segmentation. In this, they coincide with the theory formulated by Bowen and HaNson for a case of gynandromorphism in Artemia salina, but unlike these authors, they admit the possibility of a hormonal action in the male on certain tissues of the female, resulting in the masculinisation of the gonad which is geneticaly female.

\section{UBER EIN AUFTRETEN VON NATURLICHEM GYNANDROMORPHASM BEI CHIROCEPHALUS DIAPHANUS PREV.}

[Crustaceen, Branchiopode]

In diesem Aufsatz, beschreiben die Autoren ein Auftreten von bilateralem natürlichem Gynandromorphismus bei Chirocephalus diaphanus. Sie interpretieren diese sexuelle Abweichung als das Ergebnis einer zufälligen Chromosomenabänderung, die während der ersten Segmentationsteilung des Eies statt fände. Hierin stimmen Sie mit der Theorie 
von Bowen und Hanson, die sich auf einen Fall von Gynandromorphismus bei Artemia salina bezieht überein, aber entgegen der Heinung dieser Autoren, halten Sie einen Hormone einfluss des männlichen Teils auf gewesse Gewebe im weiblichen Anteil für möglich, was die Vermännlichung der genetisch weiblichen Gonade zur Folge hat.

\section{TRAVAUX GITÉS}

Batesdent (M. L.). 1964. - Recherches sur la sexualité et le déterminisme des caractères sexuels d'Asellus aquaticus L. (Crustacé Isopode). Thèse Sci. nat., Nancy, $231 \mathrm{p}$.

Banta (A. M.) et Coll. (d'après Bowen et Hanson). 1939. - Studies on the Physiology, Genetics and Evolution of some Cladocera. Carnegie Inst. Wash. Publ., 513.

Bowen (S. T.). 1963. - The genetics of Artemis salina. II. White eye, a sex-linked mutation. Biological Bull., 124 (1) : 17-23.

Bowen (S. T.), Hanson (J.). 1962. - A gynandromorph of the brine Shrimp, Artemia salina. Genetics, 47 (3) : 277-280.

Brust (R. A.). 1966. - Gynandromorphs and intersexes in Mosquitoes (Diptera : Culicidae). Canadian Journ. Zool., 44: 911-921.

Dalens (H.). 1966. - Intersexualité dans le genre Chaetophiloscia (Isopoda, Oniscoidea, Oniscidae). C. R. Acad. Sci., Paris, 262, série D : 1283-85.

Gissler (C. E.). 1881. - Description of hermaphroditic Phyllopod crustacean (Eubranchipus). Amer. Naturalist., 15 : 136-139.

Herrick (F. H.). 1896, in Chace (F. A.) Jn et Moore (G. M.). 1959. A bicolored gynandromorph of the lobster Homarus americanus. Biol. Bull., 116 : 226-231.

PAi (S.). 1949. - Sexuelle Stufen Chromosomengrösse der Keimzellen von Chirocephalus nankinensis. Exper. Cell. Research. N. Y., suppl. 1 : 143-145.

Veillet (A.). 1945. - Recherches sur le parasitisme des Crabes et Galathées par les Rhizocéphales et les Epicarides. Ann. Inst. Ocean. Monaco, 22 : 193-341.

(Laboratoire de Zoologie - Biologie animale, Université de Tunis.) 